\title{
The Signal Averaged P-Wave Is Able to Indicate the Clinical State of Atrial Fibrillation Disease
}

\author{
Matthias D Zink ${ }^{1,2}$, Stef Zeemering ${ }^{1}$, Martijn Gilbers ${ }^{1}$, Laurent Pison ${ }^{1}$, Ulrich Schotten ${ }^{1}$ \\ ${ }^{1}$ Cardiovascular Research Institute Maastricht (CARIM), Physiology, Maastricht, the Netherlands \\ ${ }^{2}$ RWTH University Aachen, Internal Medicine I, Cardiology, Pulmonology \& Vascular Medicine, \\ Aachen, Germany
}

\begin{abstract}
Atrial remodeling in patients suffering of atrial fibrillation is related to the stage of disease. During sinus rhythm, the P-wave is a surrogate for atrial electrical activation, but routine 12-lead electrocardiogram (ECG), may not be ideally suited to reflect the atrial electrical activity. In previous work we identified lead position with higher sensitivity for changes in P-wave morphology. In this study we added three novel lead positions to the 12lead ECG to determine different stages of atrial fibrillation. By means of our novel lead positions and calculations of a signal averaged ECG we were able to describe differences of $P$-wave morphology for patients with atrial fibrillation compared to a reference cohort and correlated these to the stages of disease for paroxysmal and persistent atrial fibrillation.
\end{abstract}

\section{Introduction}

Atrial fibrillation is an atrial arrhythmia with high prevalence that increases with age and comorbidities [1]. Patients suffering of atrial fibrillation are at increased risk for stroke and worsening of heart failure which increase morbidity and mortality [2]. In clinical routine, the stage of atrial fibrillation disease is defined by its persistence which is used as guidance for treatment decisions. Early stages or self-terminating atrial fibrillation are named paroxysmal, later and non self-terminating atrial fibrillation stages are named persistent [3]. However, clinical determination of the stage of atrial fibrillation is often difficult but has crucial impact on treatment. Thus, it would be desirable to identify features in electrocardiogram (ECG) related to the stage of atrial fibrillation disease.

The atrium of patients suffering of atrial fibrillation is affected by structural and conduction abnormalities related to the stage of atrial fibrillation disease [4,5]. The P-wave, a surrogate for electrical atrial activity, reflects in a routine 12-lead ECG these changes poorly, because of routine used filter settings and overall low-resolution recordings. In previous work we identified locations which were able to show the atrial electrical activity more precisely than the 12-lead ECG deflections [6]. Therefore, we added three novel lead positions (A1-A3, Figure 1) to record an extended ECG with a detailed P-wave morphology.

The aim of this investigation was to characterize the Pwave changes related to the stage of atrial fibrillation disease.

\section{Methods}

The participants were recruited from the AFAB registry (NCT03075930). We included 190 participants with history of atrial fibrillation scheduled for an ablation of atrial fibrillation. As a reference cohort without atrial fibrillation we included 57 patients of the RACE V Work Package 5 study (NCT03124576) scheduled for elective open chest surgery, mainly but not exclusively coronary revascularization. This cohort without atrial fibrillation is labeled as "reference" cohort in the text. An extended ECG was recorded at admission to the hospital in both groups. For this study we selected 117 patients with sinus rhythm in their admission ECG in the atrial fibrillation group. For the reference group we excluded patients with a history of atrial fibrillation, and heart failure and age matched 30 patients to our atrial fibrillation group.

Table 1 Reference group without history of atrial fibrillation compared to atrial fibrillation patients. AF: atrial fibrillation

\begin{tabular}{lccl}
\hline & $\begin{array}{c}\text { reference } \\
\text { Mean } / \%(\mathbf{N})\end{array}$ & $\begin{array}{c}\mathbf{A F} \\
\text { Mean } / \%(\mathbf{N})\end{array}$ & $\boldsymbol{P}$ \\
\hline Age [years] & $66 \pm 8$ & $66 \pm 8$ & 0.14 \\
Gender [male] & $87 \%(26)$ & $60 \%(70)$ & \\
Height [cm] & $174 \pm 7$ & $175 \pm 10$ & 0.27 \\
Weight [kg] & $86 \pm 14$ & $85 \pm 17$ & 0.31 \\
\hline
\end{tabular}

\subsection{Extended ECG lead positions}

In previous work we identified lead positions on the 
chest with high sensitivity for $\mathrm{P}$-wave complexity in persistent atrial fibrillation [6] and differences between patients with paroxysmal atrial fibrillation compared to a group without atrial fibrillation. Based on these findings we added 3 novel atrial lead positions (Figure 1).

- A1: Cranial end of sternum, beneath jugulum

- A2: $8 \mathrm{~cm}$ above V6 mid-axillary line

- A3: Same height as A2, $4 \mathrm{~cm}$ medial of posterior axillary line
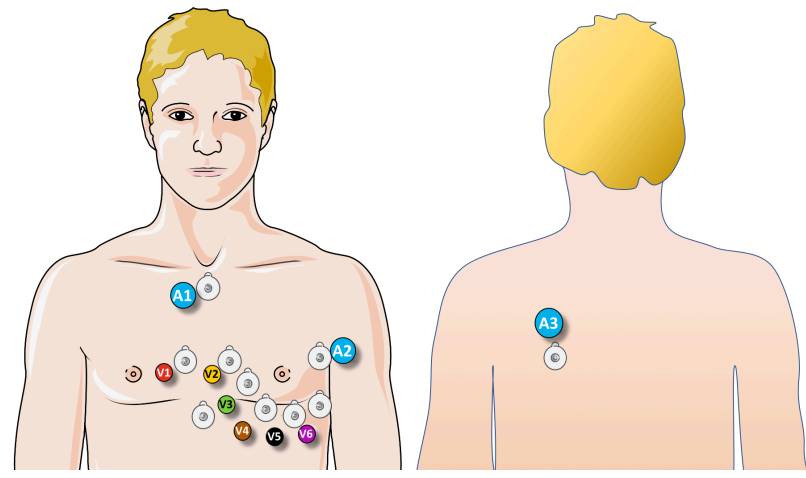

Figure 1 12-lead ECG with additional atrial lead positions $(A 1-A 3)$ to record an extended $E C G$

\subsection{Computing a signal averaged ECG}

For routine clinical use, a 12-lead ECG is mainly recorded with limited sampling frequency and extensive post processing of acquired signal is performed, e.g. baseline correction and $40 \mathrm{~Hz}$ low pass filtering.

In this study, we recorded an extended ECG with a commercially available CAM-USB ECG device (GE Healthcare) with a sampling frequency of $500 \mathrm{~Hz}(150 \mathrm{~Hz}$ low pass filter), for at least 5 minutes. Due to this long recording time, each ECG signal consists of hundreds of heart beats. By aligning and averaging the P-waves of the recorded heart beats, we obtained a high-resolution signal averaged P-wave (Figure 2) using custom developed software (Matlab 2017 R2, Mathworks). Apart from baseline correction, no additional filtering or postprocessing was performed. The signal averaged $\mathrm{P}$-wave was analyzed for duration, amplitude, terminal force in $\mathrm{V} 1$, area and complexity in the novel lead locations A1-A3, in lead II and V1.

- duration: begin to end of the P-wave measured in milliseconds (begin and end marked manually)

- amplitude: maximum to minimum peak measured in millivolt

- terminal force: area from zero-crossing of the biphasic P-wave in V1 to negative till the end of the P-wave

- $\quad$ area: enclosed area from begin to end of the P-wave
- $\quad$ complexity [7]: the number of significant changes in P-wave direction

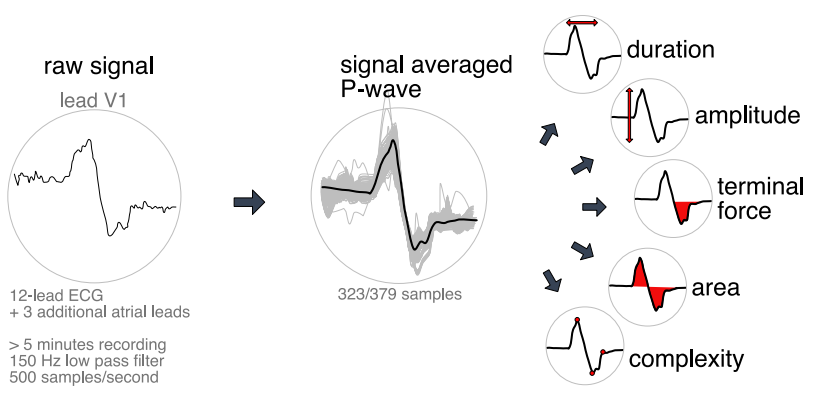

Figure 2 After the ECG signal was recorded, our algorithm identified each heartbeat and calculated a signal averaged $P$ wave. The resulting $P$-wave was analyzed for duration, amplitude, terminal force in V1, area and complexity.

\section{Results}

Mean age of participants in the atrial fibrillation group was $64 \pm 8$ years, $60 \%$ (70) were male (Table 1 ).

Table 2 Baseline parameters atrial fibrillation cohort. AF: atrial fibrillation; $L V E F$ : left ventricular ejection fraction; $L A$ : left atrium; $A F$ : atrial fibrillation

\begin{tabular}{lc}
\hline & Mean \pm SD $/ \%(\mathbf{N})$ \\
\hline AF paroxysmal & $61 \%(71)$ \\
persistent & $39 \%(46)$ \\
Ablation cryoballon & $64 \%(75)$ \\
radiofrequency & $36 \%(42)$ \\
First ablation & $81 \%(95)$ \\
LVEF [\%] & $57 \pm 8$ \\
LA diameter [mm^2] & $43 \pm 5$ \\
volume [ml] & $88 \pm 24$ \\
$\mathrm{CHA}_{2} \mathrm{DS}_{2}$ VASc Score & $2 \pm 2$ \\
\hline
\end{tabular}

\subsection{Cohort ECG features}

P-wave dispersion and PQ time showed no significant differences between the reference and the atrial fibrillation cohort. P-wave duration (Figure $3 \mathrm{~A}$ ) and terminal force in V1 (Figure 3 B) were able to significantly discriminate between patients with atrial fibrillation and those without (duration: $\mathrm{p}=0.034$; terminal force in $\mathrm{V} 1$ : $\mathrm{p}=0.018$ ). However, both parameters were not able to show significant differences between paroxysmal and persistent atrial fibrillation patients. The complexity of the $\mathrm{P}$-wave in V1 showed changes related to the stage of disease compared to the reference cohort but not between paroxysmal and persistent atrial fibrillation (Figure 4). The other leads showed trends without significance for 
complexity.

The parameter and lead combination for our novel lead locations A1, A2 and lead II with area and amplitude were able to show significant differences related to the stage of atrial fibrillation. They could also show significant results for atrial fibrillation compared to the reference cohort and for the subgroup paroxysmal atrial fibrillation compared to the reference cohort (amplitude A1: $\mathrm{p}=0.001$; amplitude lead II: $p<0.001$ respectively). Figure 5 shows an example for P-wave amplitude in the novel lead location A1.
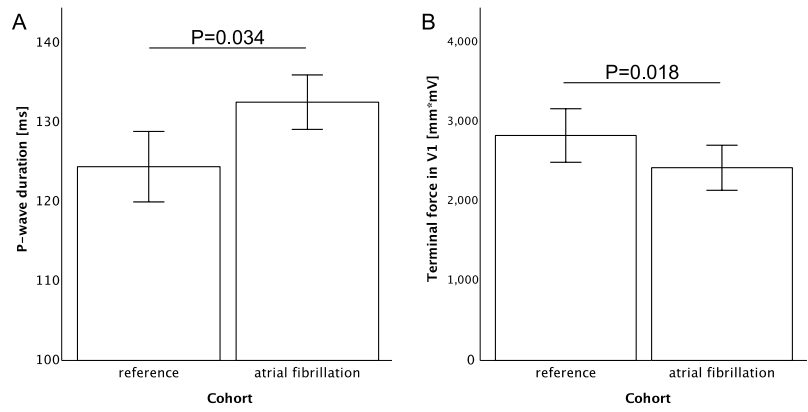

Figure 3 Duration (A) and terminal force in V1 (B) showed significant differences between reference and atrial fibrillation cohort. However, they were not able to distinguish between paroxysmal and persistent atrial fibrillation
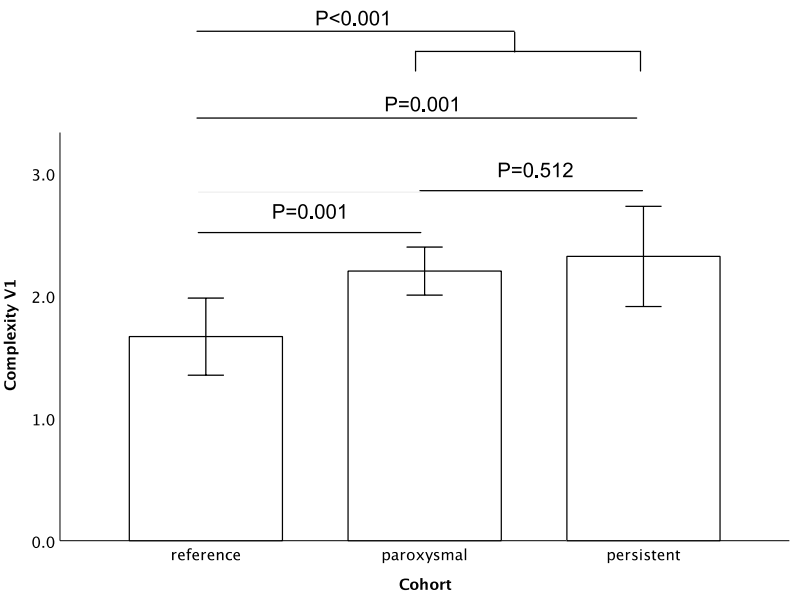

Figure 4 Complexity in V1 showed significant differences between the reference cohort and paroxysmal as well as persistent atrial fibrillation cohort. There was no significant difference between paroxysmal compared to persistent atrial fibrillation cohort.

\subsection{Identification of patients with a history of atrial fibrillation}

The area under the receiver-operator curve (ROC) for $\mathrm{P}$-wave area (in lead A1 and V1), amplitude (A1 and V1) as well as complexity (V1) showed a significant moderate to good prediction of atrial fibrillation (Table 3).

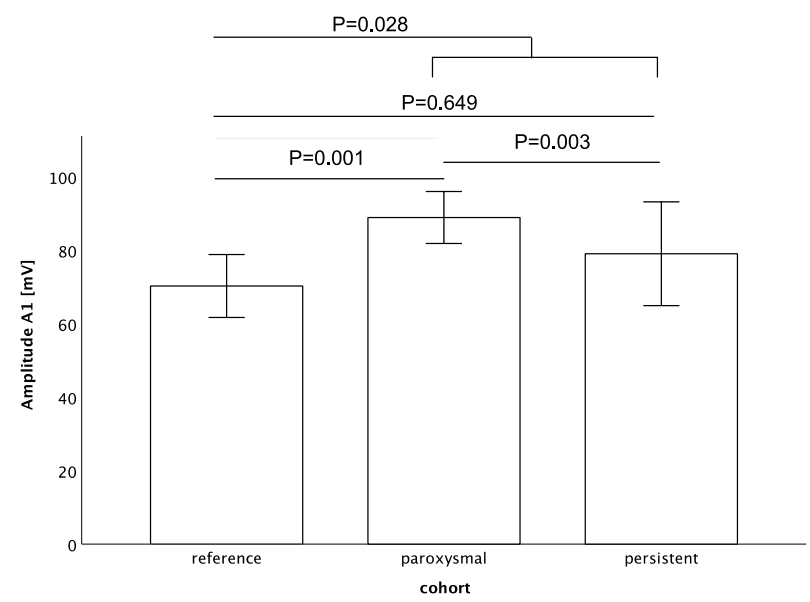

Figure 5 Novel lead position A1 (shown here) and A2 and lead II were the only parameter lead combination who could show significant differences between paroxysmal and persistent atrial fibrillation, related to the stage of atrial fibrillation disease. Amplitude in lead AI and II were also able to show significant differences between reference and atrial fibrillation cohort.

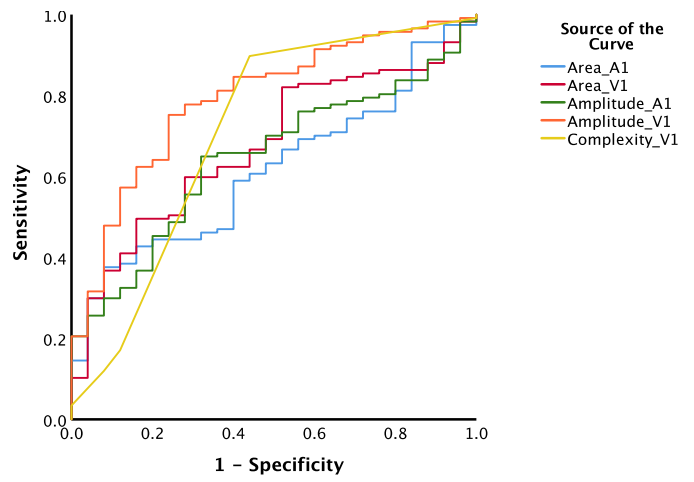

Figure 6 ROC analysis for prediction of atrial fibrillation

Table 3 Prediction of atrial fibrillation. AUC: area under the curve

\begin{tabular}{lccc}
\hline & AUC & Sensitivity & Specificity \\
\hline Area A1 & 0.62 & $63 \%$ & $52 \%$ \\
Area V1 & 0.67 & $69 \%$ & $52 \%$ \\
Amplitude A1 & 0.64 & $70 \%$ & $52 \%$ \\
Amplitude V1 & 0.79 & $78 \%$ & $72 \%$ \\
Complexity V1 & 0.71 & $90 \%$ & $56 \%$ \\
\hline
\end{tabular}

\section{Discussion}

Signal averaged P-waves combined with novel atrial lead locations provide a more sophisticated extraction of $\mathrm{P}$-wave features compared to routine 12-lead ECG. 
In previous work we identified locations cranial to the precordial leads more sensitive to P-wave morphology. In transversal view the precordial leads $(\mathrm{V} 1-\mathrm{V} 6)$ in routine 12-lead ECG describe a plane, following the longitudinal axis of the ventricles. Our idea was to use a second, more cranial plane through the atrium with our new lead positions. By combining these lead locations by means of signal averaged $\mathrm{P}$-wave calculations we were able to discriminate between the reference and the atrial fibrillation cohort, and within the atrial fibrillation cohort related to the stage of atrial fibrillation disease. New atrial lead locations showed measurements in line with the compared leads of routine 12-lead ECG. Furthermore, they offered additional information to P-wave morphology performing better in discriminating stages of atrial fibrillation than with the analysed leads of standard 12channel recordings.

An important aspect of our findings is the superiority of area, amplitude and complexity as parameters for P-wave morphology in contrast to duration to determine changes in P-wave related to atrial fibrillation. Our novel lead locations, in particular lead A1 and A2 were sensitive towards differences in the cohorts and supplement the existing leads. The combination of novel lead locations combined with means of signal averaged ECG may therefore potentially improve diagnosis and treatment of atrial fibrillation. Screening for subclinical paroxysmal atrial fibrillation needs the presence of an ongoing arrhythmia during the ECG recording. In particular in paroxysmal atrial fibrillation disease the presence of ongoing arrhythmia is rare, but this cohort seems to be already endangered for embolic stroke [8]. With a more sophisticated analysis of the P-wave, patients with ECG features indicative of a history of atrial fibrillation may be identified during sinus rhythm and monitored more closely. After diagnosis of atrial fibrillation, treatment is guided by clinical stage of atrial fibrillation disease, considering drug treatment, decision for ablation and ablation technique. However, in clinical routine discrimination between paroxysmal and persistent atrial fibrillation has a tremendous overlap. Therefore, we think the additional information of our extended ECG may help to guide physicians in their therapy decisions.

Importantly, in this study we used a commercial available ECG device; the additional leads may be recorded simultaneously or consecutively. The used filter and recording settings are available in many ECG devices already in clinical use. By a semiautomatic approach in our algorithm an analysis of an extended ECG can be done by trained persons in less than 5 minutes without harm for the patient.

\section{Conclusion}

We conclude that the combination of our novel atrial lead positions with signal averaged P-waves allows for more sophisticated ECG feature extraction. Our parameter and lead combinations were able to discriminate the reference ECG from the atrial fibrillation cohort, as well as to distinguish between paroxysmal and persistent atrial fibrillation cohort the stage of disease significantly.

\section{Acknowledgements}

This work was supported by a grant from the Dutch Heart Foundation [CVON2014-09, RACE V: Reappraisal of Atrial Fibrillation: Interaction between hyperCoagulability, Electrical remodeling, and Vascular Destabilisation in the Progression of AF], a grant from the European Union [CATCH ME-GAP-633196], and a grant from ERACoSysMed [SysAFib, No. 645782].

\section{References}

[1] Bjorck S, Palaszewski B, Friberg L, Bergfeldt L. Atrial fibrillation, stroke risk, and warfarin therapy revisited: a population-based study. Stroke 2013;44:3103-8.

[2] Andersson T, Magnuson A, Bryngelsson IL, et al. All-cause mortality in 272,186 patients hospitalized with incident atrial fibrillation 1995-2008: a Swedish nationwide long-term casecontrol study. Eur Heart J 2013;34:1061-7.

[3] Kirchhof P, Benussi S, Kotecha D, et al. 2016 ESC Guidelines for the management of atrial fibrillation developed in collaboration with EACTS. Eur Heart J 2016;37:2893-962.

[4] Schotten U, Ausma J, Stellbrink C, et al. Cellular mechanisms of depressed atrial contractility in patients with chronic atrial fibrillation. Circulation 2001;103:691-8.

[5] Nguyen BL, Fishbein MC, Chen LS, Chen PS, Masroor S. Histopathological substrate for chronic atrial fibrillation in humans. Heart Rhythm 2009;6:454-60.

[6] Zeemering S, Lankveld T, Bonizzi, et al. Principal Component Analysis of Body Surface Potential Mapping in Atrial Fibrillation Patients Suggests Additional ECG Lead Locations. Computing in Cardiology 2014; 41:893-896.

[7] Potse M, Lankveld TA, Zeemering S, et al. P-wave complexity in normal subjects and computer models. J Electrocardiol 2016;49:545-53.

[8] Vanassche T, Lauw MN, Eikelboom JW, et al. Risk of ischaemic stroke according to pattern of atrial fibrillation: analysis of 6563 aspirin-treated patients in ACTIVE-A and AVERROES. Eur Heart J 2015;36:281-7a

Address for correspondence.
Matthias Zink
Cardiovascular Research Institute Maastricht (CARIM),
Department of Physiology
Universiteitsingel 50
6229 ER Maastricht
the Netherlands
m.zink@maastrichtuniversity.nl

\author{
Matthias Zink \\ Cardiovascular Research Institute Maastricht (CARIM), \\ 6229 ER Maastricht \\ m.zink@maastrichtuniversity.nl
}

